\title{
Dynamical Evolution of Elliptical Galaxies with Central Singularities
}

\author{
David Merritt and Gerald D. Quinlan \\ Department of Physics and Astronomy, Rutgers University, New Brunswick, NJ 08855 \\ Rutgers Astrophysics Preprint Series No. 212
}

\begin{abstract}
We study the effect of a massive central singularity on the structure of a triaxial galaxy using $N$-body simulations. Starting from a single initial model, we grow black holes with various final masses $M_{h}$ and at various rates, ranging from impulsive to adiabatic. In all cases, the galaxy achieves a final shape that is nearly spherical at the center and close to axisymmetric throughout. However, the rate of change of the galaxy's shape depends strongly on the ratio $M_{h} / M_{g}$ of black hole mass to galaxy mass. When $M_{h} / M_{g} \lesssim 0.3 \%$, the galaxy evolves in shape on a timescale that exceeds $10^{2}$ orbital periods, or roughly a galaxy lifetime. When $M_{h} / M_{g} \gtrsim 2 \%$, the galaxy becomes axisymmetric in little more than a crossing time. We propose that the rapid evolution toward axisymmetric shapes that occurs when $M_{h} / M_{g} \gtrsim 10^{-2}$ provides a negative feedback mechanism which limits the mass of central black holes by cutting off their supply of fuel.
\end{abstract}

\section{Introduction}

Massive compact objects appear to be ubiquitous components of galactic nuclei (Kormendy \& Richstone 1995). While these mass concentrations need not be black holes - current observations fail by orders of magnitude to resolve the Schwarzschild radius there is a growing concensus that the black hole model is reasonable and, in many ways, simpler and more compelling than its alternatives. Particularly attractive is the possibility that we are observing the black holes that once powered quasars and that still provide the energy source of active galactic nuclei. This hypothesis is at least crudely consistent with the observed properties of quasars and AGN; in particular, quasar luminosities imply accumulated masses of order $10^{8} \mathcal{M}_{\odot}$ or more per galaxy (Soltan 1982), in reasonable agreement with the masses inferred from the nuclear kinematics of nearby galaxies. The 
strongest of the current black hole candidates have masses ranging from $\sim 10^{6.3} \mathcal{M}_{\odot}$ in the case of the Milky Way to $10^{9.5} \mathcal{M}_{\odot}$ in the case of M87; expressed as a fraction $M_{h} / M_{g}$ of the stellar mass of their host galaxies, black hole masses are more nearly constant, ranging from $\sim 0.02 \%$ to $\sim 2 \%$ (Kormendy \& Richstone 1995).

Central singularities containing such a large fraction of a galaxy's mass should have a substantial influence on the dynamics and evolution of the surrounding stars and gas. Peebles (1972) and Young (1980) noted that the growth of a black hole in a pre-existing stellar core would pull the surrounding stars into a power-law density cusp. Power-law cusps are now known to be universal components of early-type galaxies Gebhardt et al. 1996), although they are often found to be less steep than the $\rho \propto r^{-3 / 2}$ dependence that follows from adiabatic growth of a black hole growth in an isothermal sphere (Merritt \& Fridman 1995). Another consequence of the slow growth of a black hole is a polarization of the stellar velocity field: orbits of stars near the black hole become slightly more circular as a consequence of its growth (Young 1980; Goodman \& Binney 1984). Such a polarization may have been observed in M87 (Merritt \& Oh 1997).

Both of these dynamical processes were first studied in the context of spherical galaxy models, and both are predicted to strongly influence the stellar distribution only within the radius of influence of the black hole, $r_{h}=\sqrt{G M_{h} / \sigma^{2}}$, where $\sigma$ is the velocity dispersion of the stars in the nucleus. However, the influence of a massive central singularity in a non-axisymmetric galaxy can extend far beyond $r_{h}$. Many of the orbits in triaxial potentials are boxes, orbits that pass arbitrarily close to the center after a sufficiently long time. Gerhard \& Binney (1985) argued that the scattering and redistribution of box orbits by a central black hole would cause at least the inner parts of a triaxial galaxy to become rounder or more axisymmetric. Norman, May \& van Albada (1985) performed N-body integrations to follow the evolution of a triaxial galaxy as a central point mass was grown. While they confirmed in a general way the predictions of Gerhard \& Binney (1985), the small number of particles in their simulations, coupled with the relatively large softening lengths that they were forced to adopt, made it difficult to interpret their results in an unambiguous way.

In this paper, we present an $N$-body study of the influence of a growing black hole on a pre-existing triaxial galaxy. Our study benefits to a great degree from advances in the art of $N$-body simulation since the time of Norman, May \& van Albada's (1985) pioneering study. Our computer code, described in Section 2, employs individual time steps for the particles. In this way we are able to accurately follow the stellar trajectories over a great range of spatial scales, to well within the radius of influence of the black hole. We also assign different masses to particles with different binding energies in order to increase the 
effective resolution near the black hole. Our integrations employ enough particles, $\sim 10^{5}$, that collisional relaxation effects are negligible.

Our results (Sections 3,4) are in qualitative agreement with the predictions of Gerhard \& Binney (1985) and others: the black hole causes the initially triaxial galaxy to become rounder near the center and more axisymmetric throughout. But we find that the evolution toward axisymmetry is nearly complete; i.e. the model reaches an equilibrium state that differs only negligibly from (oblate) axisymmetry over a wide range in radii. Furthermore we find that the timescale over which the galaxy's shape evolves is strongly dependent on the mass of the black hole. When $M_{h} / M_{g}$ increases above $\sim 2 \%$, the evolution to an axisymmetric state takes place in a time not much longer than the crossing time; while for $M_{h} / M_{g} \lesssim 0.01$, evolution requires of order $10^{2}$ crossing times or longer. We argue, based on test-particle integrations, that the sudden decrease in the galaxy response time when $M_{h} / M_{g}$ exceeds 0.02 is a consequence of a transition to global stochasticity in the phase space of boxlike orbits.

Our results suggest that there is a characteristic mass fraction, of order $M_{h} / M_{g} \approx 10^{-2}$, at which a central black hole will cause its host galaxy to evolve rapidly to an axisymmetric state. This mass fraction is close to the maximum value of $M_{h} / M_{g}$ observed in nearby galaxies: several galaxies have $M_{h} / M_{g} \approx 10^{-2}$, and the current record-holder, NGC 3115, has $M_{h} / M_{g} \approx 0.02$. We suggest that this agreement is more than a coincidence. The fueling of massive black holes in active galactic nuclei and quasars requires matter to be channeled into the nucleus from large distances, and gravitational torques resulting from a bar-shaped or triaxial mass distribution are an efficient way to accomplish this. The masses of nuclear black holes may be regulated by a negative-feedback mechanism that shuts off the supply of fuel to the nucleus once $M_{h} / M_{g}$ exceeds roughly the critical value found here (Section 5).

\section{Numerical Techniques and Initial Conditions}

The $N$-body code used here was a hybrid, combining parts from the mean-field code of Hernquist \& Ostriker (1992) and the NBODY programs of Aarseth (1994). The equation of motion for the stars was

$$
\ddot{\mathbf{r}}_{k}=-\frac{M(t) \mathbf{r}_{k}}{\left(r_{k}^{2}+\epsilon^{2}\right)^{3 / 2}}-\sum_{n, l, m} A_{n l m} \nabla \Phi_{n l m} ;
$$

the black hole, of mass $M(t)$, remained fixed at the origin. The first term of Equation 1 describes the interaction of the stars with the black hole, while the second represents the star-star interactions. The latter were computed via a basis-function expansion of the 
potential, with coefficients $A_{n l m}$ that were periodically updated. The indices $n$ and $l$ run from 0 to $n_{\max }$ and 0 to $l_{\max }$; we used $n_{\max }=16$ radial basis functions and $l_{\max }=4$ angular basis functions. Some test experiments with larger $n_{\max }$ and $l_{\max }$ produced very similar results. Stars were advanced with individual timesteps (sometimes differing by as much as a factor of $10^{6}$ ) using Aarseth's (1994) fourth-order NBODY1 integrator. The timesteps were updated after each step by a function involving the force and the first three time derivatives of the force, multiplied by the square root of an accuracy parameter $\eta$. We used $\eta=0.01$, smaller than the value recommended by Aarseth $(\eta=0.02-0.03)$. The coefficients $A_{n l m}$ for the potential expansion were updated at fixed time intervals $\Delta t=0.0005$, after the coordinates of the stars were predicted to a common time.

The same initial model was used for all of the integrations described here. We generated a steady-state, triaxial, nonrotating $N$-body galaxy containing $1.1 \times 10^{5}$ particles as follows.

1. A random, spherical distribution of $10^{4}$ particles with density profile $\rho \propto r^{-1.5}$, $0<r<2$ was set up. The particles were initially at rest. The combined mass of these particles was set equal to one; fixing $G=1$ thus determined the model units.

2. This configuration was evolved forward with the $N$-body code, with $M_{h}=0$, until a time $t=50$. During this time, the model collapsed and re-expanded, forming in the process a nearly steady-state, triaxial bar as in Aguilar and Merritt (1990) and Cannizzo \& Hollister (1992).

3. The particles in the equilibrium triaxial model were sorted by binding energy into 10 groups. Each particle in the most-bound group was replaced by 10 particles, whose coordinates were computed by integrating the triaxial model for 2 additional times units and storing the positions and velocities at 10 equally-spaced times. In the same way, the particles in the next binding-energy group were replaced by 9 particles each, then by 8 , etc.; particles in the least-bound group were not subdivided. The total mass of the model was left unchanged.

This scheme for subdividing particles was designed to increase the resolution of the $N$-body code very near the center, where the black hole produces a cusp in the density profile.

4. Each of the $5.5 \times 10^{4}$ particles so generated was then replaced by two particles, one at the original position $(\mathbf{r}, \mathbf{v})$ and one at $(-\mathbf{r},-\mathbf{v})$. This procedure guaranteed that the center of mass of the model was initially at the origin and that the model had no net momentum.

5. The resulting set of $1.1 \times 10^{5}$ particles was evolved for another 20 time units to 
ensure that the model had reached equilibrium. The configuration obtained at the end of this integration was defined as the initial model.

The initial model is a triaxial body with a short-to-long axis ratio $c / a$ of approximately 0.5 , and a "triaxiality index" $T=\left(a^{2}-b^{2}\right) /\left(a^{2}-c^{2}\right) \approx 0.6$. The model is strongly peanut-shaped as seen from the intermediate axis (Figure 5), a common feature of $N$-body bars. The spherically-symmetrized properties of the model are shown in Figure 3. The density profile exhibits a constant-density core of radius $r_{c} \approx 0.08$, and a half-mass radius of 0.48. The particle motions are approximately isotropic in the core, and become increasingly radial in the envelope (Figure 3c) - a relic of the radial collapse. Table 1 lists some of the global properties of the initial model. Roughly $89 \%$ of the mass in the original spherical cloud remained bound after the collapse. (Thus the bound mass of the initial triaxial model is 0.89 in the units adopted here.) Approximating the model as spherically symmetric, the full period of a circular orbit at the radius containing one-half of the bound mass is $\sim 2.9$.

Figure 1 shows that the initial model is close to equilibrium. Plotted there is the time dependence of the axis ratios of the most-bound $2 \%, 10 \%$ and $50 \%$ of the particles, defined as the axis ratios of a homogeneous spheroid with the same moment-of-inertia tensor as the particles. Over an elapsed time of 50 units - roughly 17 half-mass orbital periods - there is no discernible change in the shape of the model. The radii containing $1 \%, 2 \%$, etc. of the total mass were likewise found to remain essentially constant over this interval.

The black hole was grown by increasing the mass at the center of the model according to the relation

$$
\begin{aligned}
M(t) & =M_{h} \tau^{2}(3-2 \tau), \quad \tau \leq 1 \\
& =M_{h}, \quad \tau>1
\end{aligned}
$$

with $\tau=t / t_{\text {grow }}$ (Figure 2). Various values for $M_{h}$ and $t_{\text {grow }}$ were used, as discussed below. Because the mass of stars bound to the initial model is 0.89 in our units, the final black hole mass - expressed as a fraction of the bound mass of the galaxy - is approximately $1.12 M_{h}$.

The black hole softening length $\epsilon$ of Equation (1) was computed via the relation

$$
v_{\epsilon}=8=\sqrt{G M_{h} / \epsilon}
$$

in other words, the softening length was chosen such that a typical star at a distance $\epsilon$ from the black hole would have an orbital velocity of 8 in model units. For comparison, the central velocity dispersion of the initial model is approximately 1.2 (Figure $3 \mathrm{~b}$ ). The softening length - which is a function of $M_{h}$ - is given in Table 1 for the three values of $M_{h}$ used here. Our adopted softening length was always much smaller than the radius of 
influence $r_{h}$ of the black hole. By choosing $\epsilon \ll r_{h}$, we were able to accurately reproduce the formation of a steep stellar cusp as the black hole grew.

The $N$-body code conserved the total energy of the model to $\Delta E / E \approx 0.002$ over 50 time units with no black hole. Once the black hole had grown, energy was typically conserved to $\Delta E / E \approx 0.02$ over the same time interval.

\section{Results}

The growth of the black hole caused the initially triaxial model to become nearly spherical near the center. We therefore begin by describing the spherically-symmetrized structure of the models at late times, and we compare our results with those of earlier modelling that imposed spherical symmetry. Here and below, smooth estimates of spatially-dependent properties were computed from the discrete particle coordinates via nonparametric function estimators, either adaptive kernels or smoothing splines (Wahba 1990; Green \& Silverman 1994).

\subsection{Spherically Symmetrized Profiles}

Figure 3a shows the dependence of the central density profile at $t=40$ on $M_{h}$, for three integrations with $t_{\text {grow }}=15$. The initial model has a small core, with radius $r_{c} \approx 0.08$. This core is replaced by a cusp following the growth of the black hole in all three models; within the cusp, the dependence of density on radius is well approximated as a power law, $\rho \sim r^{-\gamma}$ with $\gamma \approx 2$. In the runs with the smaller black holes, $M_{h}=0.003$ and 0.01 , the signature of the core persists even after growth of the black hole in the form of an inflection in the density profile at $r \approx r_{h}$.

Quinlan, Hernquist \& Sigurdsson (1995) computed the evolution of the stellar distribution function $f\left(E, L^{2}\right)$ of a spherical model as the mass of a central singularity was slowly increased. In models with cores, the growth of the black hole produced profiles similar to those of Figure 3a (e.g. their Fig. 3); the logarithmic slope of the central density cusp was usually close to -2 and only weakly dependent on the initial density profile. Their results were confirmed by Sigurdsson, Quinlan \& Hernquist (1995) in spherically-symmetric $N$-body integrations.

The velocity dispersion profile (computed by assuming a spherical velocity ellipsoid) also exhibits a cusp after the growth of the black hole (Figure 3b), with $\sigma \sim r^{-1 / 2}$, as expected for particles moving in response to an inverse-square force law. The velocity 
dispersion in the initial model falls slightly toward the center, a common feature in models with steeper-than-isothermal central density profiles (e.g. Binney 1980). This drop is retained following the growth of the smallest black hole, $M_{h}=0.003$, and even for $M_{h}=0.03$ the velocity dipsersion profile exhibits a mild inflection at $r \approx r_{h}$. These results are again similar to those of Quinlan, Hernquist \& Sigurdsson (1995) (e.g. their Fig. 4) and Sigurdsson, Quinlan \& Hernquist (1995).

Adiabatic growth of a central point mass in an initially constant-density core is expected to circularize the stellar orbits slightly (Young 1980; Goodman \& Binney 1984). The effect is subtle, since orbital eccentricities are left essentially unchanged by slow changes in the potential (Lynden-Bell 1963); the velocity polarization is a second-order effect resulting from the different character of orbits in the Keplerian and harmonic-oscillator limits. We nevertheless see the effect here (Figure 3c): the velocity anisotropy, $\beta(r)=1-\sigma_{t}^{2}(r) / \sigma_{r}^{2}(r)$, falls slightly below zero for $r \lesssim r_{h}$ in the models with $M_{h}=0.01$ and 0.03 , indicating a slight bias toward circular motions. However, $\beta$ does not drop below about -0.2 . Quinlan, Hernquist \& Sigurdsson (1995) and Sigurdsson, Quinlan \& Hernquist (1995) found similar, modest changes in $\beta$ at small radii in spherical models with comparable black hole masses.

The "observable" velocity dispersion profile is presented in Figure 3d. Here the averaging was carried out over circular rings in the plane of the sky, defined as the plane perpendicular to the intermediate axis of the model. The $r^{-1 / 2}$ velocity cusp is clearly visible in the projected profile as well.

To summarize: The slow growth of a central point mass induces changes near the center of the $N$-body model that are similar to those predicted by Young (1980), Goodman \& Binney (1984), Quinlan, Hernquist \& Sigurdsson (1995) and Sigurdsson, Quinlan \& Hernquist (1995) on the basis of spherical models. The constant-density core is replaced by an $r^{-2}$ cusp; the velocity dispersion increases as $r^{-1 / 2}$ near the black hole; and the velocity ellipsoid becomes mildly elongated in directions tangential to the radius vector. All of these changes are confined to a radius $r \lesssim r_{h}$.

\subsection{Model Shapes}

Not all effects of the central singularity are confined to small radii, however. Figure 4 illustrates the time-dependence of the model axis ratios for three integrations with $M_{h}=0.003,0.01,0.03$ and $t_{\text {grow }}=15$. Axis ratios were defined in terms of the principal axes $a \geq b \geq c$ of the homogenous ellipsoid with the same moment-of-inertia tensor as the $N$-body model. Following the usual practice, particles were first ranked according to binding 
energy and the inertia tensor was computed for various subsets, e.g. the most-bound $2 \%$, $10 \%$ etc. Figure 4 shows that the model changes shape even at large radii, out to and exceeding the half-mass radius. Near the center - at radii corresponding to the inner few percent of the mass in stars - all of the models become nearly spherical, on a timescale that is comparable to $t_{\text {grow }}$. At larger radii, both axis ratios $b / a$ and $c / a$ initially increase, but the evolution appears to slow or halt once the models approach axisymmetry, i.e. when $b / a$ comes close to one. This is seen most clearly in the integration with $M_{h}=0.03$, where the evolution is essentially complete once the black hole has reached its full mass at $t=15$. In the runs with the smaller black hole masses, axisymmetry within the half-mass radius is barely reached at the final time step and slow evolution continues until the end of the run. The evolution timescale is also a function of location in the model, i.e. longer at larger radii.

The evolution seen here toward rounder or more axisymmetric shapes is not surprising; similar evolution has been observed in a number of $N$-body studies of triaxial galaxies following an increase in the central density (Norman, May \& van Albada 1985; Udry 1993; Dubinski 1993; Barnes \& Hernquist 1996; Norman, Sellwood \& Hasan 1996). The mechanism that drives the evolution is discussed in detail below; here we note only that the evolution appears to be dependent both on the presence of a central singularity and on departures from axisymmetry, and that it slows or stops once the models are close to axisymmetric.

One nevertheless worries that the evolution seen in Figure 4 might be due in part to systematic errors in the $N$-body code. Most errors in the force computations or the time integration would tend to produce spurious evolution toward spherical symmetry. We note that the run with the largest black hole exhibits essentially no evolution once axisymmetry is reached; the model accurately maintains its highly flattened, axisymmetric shape. On the other hand, one might not expect integration errors to have much effect in axisymmetric models since angular momentum conservation would keep most particles from approaching the black hole.

The fact that the stellar cusps, once formed, persist without significant change also argues in favor of the $N$-body integrations being carried out correctly in the vicinity of the black hole. But here, too, a cautionary note is in order. The representation of the potential via a basis set (Eq. 1) is not optimal. Since the basis functions used here correspond to mass components with central cusps, the $N$-body code might tend to produce cusps or to spuriously maintain them once formed. A code that represented the radial part of $\Phi$ on a grid, with local smoothing, would be preferred (Merritt 1996) and should be applied to this problem as a check on our results. 
Although the timescale for the evolution in Figure 4 is strongly dependent on $M_{h}$, the equilibrium figures of the three models are very similar: all models tend toward axisymmetry, even at radii exceeding the half-mass radius, and the short-to-long axis ratio of the final configuration varies from $\sim 1$ at the smallest radii to $\sim 0.6$ near the half-mass radius in each of the models. Figure 5 illustrates the projected appearance of the model with the intermediate-mass black hole, $M_{h}=0.01$, at the final time step $(t=80)$, as seen from a point in the equatorial plane. The initial model is also shown for comparison, as seen from the intermediate axis. The elongation of the initial model is approximately constant with radius, and its isophotes are strongly peanut-shaped at intermediate radii - a common feature of $N$-body galaxies (though strongly non-elliptical isophotes are rare in real galaxies). The isophotes of the model with $M_{h}=0.01$ become progressively more elongated with increasing radius, although they remain rounder than those of the initial model at least until a radius of $\sim 5$, where the dynamical time is $\sim 80$, roughly equal to the integration time.

\subsection{Evolution Timescales}

Inspection of Figure 4 reveals one intriguing result. In the integration with the largest black hole, $M_{h}=0.03$, the model reaches axisymmetry at all three radii displayed $\left(r_{2}, r_{10}, r_{50}\right)$ by roughly the time the black hole has ceased growing, i.e. by $t \approx 15$. In other words, the evolution of the model's figure appears to be limited only by the growth rate of the black hole. By comparison, in the integrations with smaller black holes, $M_{h}=0.01$ and $M_{h}=0.003$, the model figure evolves at different rates at different radii, and the evolution requires much longer to reach completion - roughly 100 time units ( $\sim 30$ orbital periods) at the half-mass radius in the model with $M_{h}=0.01$, and perhaps $200-300$ time units $(\sim 80$ orbital periods) in the model with $M_{h}=0.003$. Only at the very centers of these two models - where the orbital period is a small fraction of the black hole growth time - does the shape of the model appear to evolve at a rate that is limited by the growth of the black hole. Outside of the very center, the model figure evolves on a timescale that is approximately a constant multiple (different for the two values of $M_{h}$ ) of the local orbital period.

To better understand the very rapid evolution observed in the model with $M_{h}=0.03$, we repeated this integration with three different values of the black hole growth time: $t_{\text {grow }}=0.1,5$ and 45 . The shortest of these growth times, $t_{\text {grow }}=0.1$, is small compared even with the orbital period at the center of the initial model; hence the growth of the black hole in this integration is essentially impulsive as seen by the stars. Figure 6 reveals that the model responds to the $M_{h}=0.03$ black hole about as quickly as it can; in the impulsive 
limit, the model evolves to axisymmetry at all radii on a timescale that is close to the local orbital period. For instance, the intermediate-to-long axis ratio $b / a$ determined by the $50 \%$ most-bound stars reaches unity by a time of $\sim 2.5$ when $t_{\text {grow }}=0.1$, compared to a circular orbital period of $\sim 2.9$ at the half-mass radius (Table 1 ).

These experiments suggest that the timescale on which the galaxy responds in shape to

the appearance of the black hole is a steep function of the black hole's mass. Figure 7 plots the evolution toward axisymmetry of the most-bound $50 \%$ of the stars for the three values of $M_{h}$. The curves for the two smaller black hole masses were taken from the simulations with $t_{\text {grow }}=15$, which is shorter than the apparent response time of the galaxy at the half-mass radius for these values of $M_{h}$. The curve for $M_{h}=0.03$ is from the integration with $t_{\text {grow }}=5$, short enough that the galaxy's response at the half-mass radius is not strongly limited by the black hole growth rate. Figure 7 suggests that the response time of the galaxy to the black hole drops suddenly when $M_{h}$ exceeds $\sim 10^{-2}$.

The critical mass fraction can be estimated more accurately by looking at the integration with the longer black-hole growth time, $t_{\text {grow }}=45$ (Figure 6a). Axisymmetry at all radii is reached in these integrations somewhat before the black hole has attained its final mass of 0.03 . For instance, at the radius containing $10 \%$ of the stars, $b / a \approx 1$ when $t \approx 30$, at which time the black hole mass is $\sim 0.025$, or $M_{h} / M_{g} \approx 0.027$. At the radius containing $50 \%$ of the stars, approximate axisymmetry is reached when $t \approx 40$; taking into account the orbital time at this radius suggests a critical mass fraction for transition to axisymmetry of $M_{h} / M_{g} \approx 0.028$. Thus, evolution toward axisymmetry takes place very rapidly once $M_{h} / M_{g}$ exceeds $\sim 2.5 \%$.

\section{Evolution Mechanism}

The evolution toward axisymmetric shapes seen here is an expected consequence of stochasticity in the stellar orbits, induced by the central singularity. The argument - which is not original with us - goes roughly as follows; fuller discussion may be found in the papers of Schwarzschild (1981), Norman, May \& van Albada (1985), Gerhard \& Binney (1986), Gerhard (1987), Udry \& Pfenniger (1988), Merritt \& Fridman (1996), Barnes \& Hernquist (1996), Merritt \& Valluri (1996), and Merritt (1997a,b).

1. Many of the stars in the initial, triaxial model are on regular box orbits that carry them close to the center, once per orbital period. (A regular orbit is defined as an orbit with three isolating integrals of the motion, two in addition to the energy.)

2. Most of the box orbits lose two of their three integrals of motion once the black 
hole appears, essentially because of large-angle deflections suffered during close passages to the center. (The exceptions are boxlike orbits that lie close to a stable resonant orbit that avoids the center, e.g. the $2: 1$ "banana.")

3. Trajectories defined by only one integral of motion - the energy - densely fill the region within the equipotential surface $\Phi(\mathbf{x})=E$. The time-averaged shape of such an orbit is approximately spherical.

4. Because box orbits with a variety of shapes are crucial for maintaining strongly triaxial figures, the model can not preserve its triaxial shape once the black hole appears. It begins to evolve toward more nearly spherical, or at least axisymmetric, shapes.

5. As axisymmetry is approached, the boxlike orbits disappear, since axisymmetric potentials only support tube orbits - orbits that conserve angular momentum about the symmetry axis and avoid the center. The black hole is then no longer effective at inducing stochasticity in the orbital motion, and the galaxy evolves to a steady, axisymmetric state in a few crossing times.

The evolution of the $N$-body models described above is consistent on a qualitative level with this picture. Especially compelling to us is the fact that the $N$-body models evolve in shape only when two conditions are satisfied: the model is triaxial, i.e. departs from axial symmetry; and $M_{h} \neq 0$. The initial, strongly triaxial model does not evolve (Figure 1), presumably because the box orbits which it contains are regular, or nearly so - consistent with the expected behavior of boxlike orbits in triaxial models with smooth cores (e.g. Goodman \& Schwarzschild 1981). And after the black hole is grown, the models cease evolving once axisymmetry is reached (Figures 4, 6), i.e. once the box orbits have disappeared. Furthermore, the evolution of the model's shape is rapid compared to the expected rate of two-body relaxation, nor is any evidence of collisional relaxation apparent in the test integrations of the initial model without a black hole. Taken together, these facts suggest that the evolution which we observe is being driven primarily by the interaction of boxlike orbits with the central singularity.

Further evidence in favor of this picture is provided by the timescales for evolution observed in the $N$-body models. Once a black hole is introduced, the box orbits would be expected to respond by gradually filling the phase-space volume made available to them i.e. the volume defined by the full energy hypersurface, minus those regions occupied by regular orbits, mostly tubes. This relaxation toward a uniform population of the accessible phase space is called chaotic mixing (Kandrup \& Mahon 1994), and it has a characteristic timescale that can be measured via test-particle integrations in fixed potentials. Merritt \& Valluri (1996) carried out such calculations in two triaxial models, one with a central 
density cusp, the other with a smooth core and a central point mass containing $0.3 \%$ of the mass of the model. Those authors evolved ensembles of $10^{4}$ particles started from various regions on the equipotential surface and recorded their relaxation to a steady state. Mixing timescales in both models were found to depend on the starting point of the ensemble. Ensembles located initially in the most stochastic parts of phase space - e.g. near the short axis of the figure - were found to mix most rapidly, filling much of their allowed region after just $10-30$ orbital periods. Other ensembles were found to mix more slowly, remaining trapped in limited regions of phase space for $10^{2}$ orbital periods or longer. Merritt \& Valluri (1996) suggested an average relaxation time associated with chaotic mixing in these models of $\sim 100$ orbital periods. This estimate is quite consistent with the rate at which the $N$-body model with $M_{h}=0.003$ is found here to evolve - the intermediate-to-long axis ratio changes from 0.76 to 0.9 in about 50 half-mass orbital periods (Figure 7).

Merritt \& Valluri (1996) did not extend their mixing calculations to triaxial models with larger black hole masses, nor are we aware of any other 3-D test-particle studies that could be usefully compared to our $N$-body models with $M_{h}=0.01$ and 0.03 . For this reason, we carried out new test-particle integrations in triaxial models with central point masses of various sizes. We adopted the "Imperfect Ellipsoid" model of Merritt \& Valluri (1996), which has mass distribution

$$
\rho(m)=\frac{\rho_{0} m_{0}^{2}}{\left(1+m^{2}\right)\left(m_{0}^{2}+m^{2}\right)}, \quad 0 \leq m_{0} \leq 1
$$

the central density $\rho_{0}$ is related to the total mass $M$ by $\rho_{0}=M\left(1+m_{0}\right) /\left(2 \pi^{2} a b c m_{0}^{2}\right)$. The parameter $m_{0}$ is a core radius; we chose $m_{0}=0.1 a$. The axis ratios were taken to be $c / a=0.5$ and $b / a=0.79$, similar to the values in the initial $N$-body model. To this model was added a central point mass containing various fractions $M_{h}$ of the total mass $M$; we used $M_{h}=0,0.003,0.01,0.03$, and 0.1 . Test-particle integrations were then carried out for sets of orbits with starting points distributed over the half-mass equipotential surface, as described in Merritt \& Valluri (1996).

Of greatest interest here is the configuration space volume filled by boxlike orbits over a relatively short interval of time. We accordingly integrated orbits for only 10 periods of the long-axis orbit. We found a striking result: over this short time interval, the behavior of the boxlike orbits changed suddenly as $M_{h}$ was increased (Figure 8). For $M_{h} \leq 0.01$, the boxlike orbits - particularly the thin boxes with starting points near the long axis of the figure, which are crucial for maintaining a triaxial shape - showed relatively little evidence of stochastic evolution. While often not regular, the orbits in these models filled regions quite similar in shape to the regions occupied by orbits in the model with $M_{h}=0$. By contrast, when $M_{h}$ was increased from 0.01 to 0.03 , essentially none of the boxlike orbits 
were found to maintain well-defined boxy shapes over 10 orbital periods. Instead, they quasi-randomly filled a rougly axisymmetric region, with almost no hint of "memory" from one oscillation to the next of the location of the previous turning point. The only exceptions were orbits with starting points near to the $x-z$ banana.

We speculate that the change in the character of the boxlike orbits as $M_{h}$ is increased from 0.01 to 0.03 is indicative of a transition to global stochasticity (e.g. Lichtenberg \& Lieberman 1983). In many dynamical systems, one observes a sharp transition as a parameter is varied: from stochastic motion that is closely bounded by KAM surfaces, i.e. regular orbits; to motion that is strongly interconnected over large portions of the space. In the latter regime, stochastic trajectories at a given energy are nearly indistinguishable, moving rapidly - in just a few oscillations - from one part of stochastic phase space to another. This is just the behavior illustrated in Figure 8 as $M_{h}$ is increased above 0.01.

Whether or not this interpretation is correct, Figure 8 suggests that the timescale over which stochasticity induces changes in the phase-space population of a triaxial model should drop from $\gg 10$ orbital periods when $M_{h} \lesssim 0.01$, to $\lesssim 10$ orbital periods when $M_{h} \gtrsim 0.03$. This is what we observe in the $N$-body models (Figure 7 ). We might predict an even steeper dependence of evolution rates on $M_{h}$ in the $N$-body models than in the test-particle integrations, since the $N$-body models develop a central density cusp with a strength that increases with $M_{h}$ (Figure 3a), and a cusp will itself induce stochasticity in the motion of boxlike orbits (Merritt \& Fridman 1996).

One final question concerns the end states of the $N$-body models. Their figures are remarkably close to axisymmetric. This is particularly true with regard to the integrations with the largest black hole, $M_{h}=0.03$, where $b / a$ at the final time step is statistically consistent with unity at all radii displayed in Figures 4 and 6 . The approach to axisymmetry is not as complete in the integrations with the smaller black holes, but Figure 4 suggests that a modest increase in the integration times would probably have produced a precisely axisymmetric end state for these values of $M_{h}$ as well.

One might have expected the $N$-body models to reach equilibrium without evolving so completely to axisymmetry. For instance, Merritt (1997b), in a study of the triaxial self-consistency problem, found that models with $\rho \propto r^{-2}$ central density cusps could exist in equilibrium for values of $b / a$ exceeding $\sim 0.85-0.9$. But there are a number of reasons why the $N$-body models should prefer even more axisymmetric states than the self-consistency studies would suggest. First, the $N$-body models contain both steep stellar cusps and central singularities; hence one would expect stochasticity to be more destructive of the boxlike orbits in this study than in that of Merritt (1997b). Second, the self-consistency studies are relatively crude in their treatment of the effects of the stochasticity; for instance, 
Merritt (1997b) simply eliminated orbits that gave evidence of instability after 50 orbital periods. In fact, orbital instability rates scale roughly with the local orbital frequency, making them a strong function of radius. Hence one should eliminate a larger fraction of the boxlike orbits near the center than at large radii, making it more difficult to reconstruct a self-consistent triaxial shape. Third, the rapid evolution toward spherical symmetry at the very centers of the $N$-body models must influence the behavior of orbits at larger energies, perhaps accelerating their evolution. It is conceivable that modestly triaxial equilibria do exist even with central singularities, but that our scheme of growing the black hole in a pre-existing triaxial model prevents us from arriving at these end states.

\section{Discussion}

We have found that a central singularity containing more than $2.5 \%$ the mass of its host galaxy induces a rapid transition from triaxiality to axisymmetry in the distribution of the surrounding stars, both near the center and at appreciable distances. Less massive singularities induce a more gradual evolution, but the end state is still close to axisymmetric; in fact, our results suggest that the shape of an initially triaxial galaxy containing a central singularity of mass $M_{h}$ is approximately independent of $M_{h}$. (Other features of the final state, like the central density profile, clearly do depend on $M_{h}$. The final state also presumably depends on the details of the initial model, which we did not vary). We argued that the sudden change in the rate of evolution of the galaxy's shape as $M_{h} / M_{g}$ exceeds $\sim 0.025$ is due to a transition to global stochasticity in the phase space of the boxlike orbits, orbits which are required to maintain triaxiality.

While it would be dangerous to generalize from such a small set of experiments, it seems reasonable to suppose that a sudden transition to axisymmetry would occur in most triaxial models as the mass of a central singularity is increased beyond some critical value. We discuss this hypothesis in more detail below; for the moment, we assume that such a transition generally occurs for central point masses of order $10^{-2} M_{g}$. Is there any evidence from real galaxies for such a critical mass?

It is well known that nuclear black holes often contain of order $1 \%$ the mass of their host galaxies. Figure 9 shows $M_{h} / M_{g}$ for the six galaxies with the largest black hole mass fractions. $M_{g}$ is defined in that figure in the usual (and, for this study, appropriate) way as the mass of the stellar bulge in the case of disk galaxies, and as the total stellar mass in the case of elliptical galaxies. Uncertainties in estimates of $M_{h}$ vary considerably from galaxy to galaxy; each of the black hole masses in Figure 9 is considered to be fairly accurate, with uncertainties of order $50 \%$ or less (Kormendy \& Richstone 1995). (One galaxy which 
may have a very large black hole mass fraction, NGC 4486b (Kormendy et al. 1997), has been omitted from the figure since the uncertainty in the black hole's mass is very large; in fact the kinematical data for this galaxy are consistent with $M_{h}=0$.) Figure 9 confirms a tendency for the most massive black holes to contain approximately $1 \%$ the stellar mass of their host galaxies, both in very bright (M87) and very faint (M32) galaxies. The largest, well-determined mass fraction is associated with NGC 3115, which has $M_{h} / M_{g} \approx 0.024$. The remainder of the galaxies have $M_{h} / M_{g} \lesssim 0.01$.

Based on this modest sample, it appears that nature avoids making nuclear black holes with masses exceeding $\sim 0.02 M_{g}$ - quite close to the critical mass that induces a transition to axisymmetry in our simulations. We suggest that this agreement is not a coincidence. The fueling of massive black holes in active galactic nuclei and quasars requires matter to be funneled into the nucleus from large distances, of order a kiloparsec or more (Shlosman, Begelman \& Frank 1990). Models that produce fuel locally, e.g. from a dense star cluster surrounding the black hole (Voit \& Shull 1988), generally fail to supply mass at a rate adequate to power the most luminous active nuclei. In order for infall to occur over such large scales, the angular momentum of the stars or gas must somehow be removed. Gravitational torques resulting from a bar-shaped or triaxial mass distribution are an efficient way to accomplish this (Norman \& Silk 1983), and there is some evidence that nuclear activity is uniquely associated with barlike or triaxial geometries (Adams 1977; Simkin, Su \& Schwarz 1980).

The rapid evolution toward axisymmetry that occurs when $M_{h} / M_{g}$ exceeds a critical value suggests that the growth of nuclear black holes is self-limiting. Once a black hole has accreted more than $\sim 10^{-2}$ the mass of its host galaxy, the galaxy would evolve rapidly to a nearly axisymmetric shape; the supply of fuel would effectively be cut off and the black hole would cease to grow. In this picture, nuclear black holes might be expected to often contain of order $1 \%$ the mass of their host galaxies, and their masses should never appreciably exceed this value, unless the black hole had grown in much less than a galaxy crossing time, or unless the galaxy had lost a significant fraction of its stellar mass after the black hole had formed. An additional prediction, of course, is that galaxies containing "maximal" black holes should be close to axisymmetric, at least near their centers.

Some of the galaxies in Figure 9 (NGC 3115, NGC 4258) are strongly rotating, and even the slowly-rotating ellipticals like M87 may have formed through mergers of rapidly-rotating disks. If so, their nuclear black holes presumably formed in the pre-existing disks - a very different environment from the one modelled here. Test-particle integrations in rotating barred potentials (Hasan \& Norman 1990; Hasan, Pfenniger \& Norman 1993) reveal that central mass concentrations can destroy the major families of bar-supporting 
orbits when $M_{h} / M_{g}$ exceeds a few percent - with $M_{g}$ here defined as the mass of the bulge plus disk. Self-consistent simulations of the response of a barred disk galaxy to the growth of a nuclear black hole have been carried out by Norman, Sellwood \& Hasan (1996). When motion was restricted to the plane of the disk, rapid evolution toward axisymmetry took place when $M_{h}$ exceeded about $5 \% M_{g}$. However, much of the instability resulting from central mass concentrations is associated with motion out of the principal planes (Pfenniger \& Friedli 1991; Fridman \& Merritt 1997), and there are indications (Sellwood \& Moore 1998) that bars in fully 3D disk galaxy simulations are more fragile than in two dimensions, evolving rapidly in shape when the black hole mass fraction exceeds only $\sim 1.5 \%$. Although a great deal of work remains to be done, it seems plausible that the critical black hole mass fraction for transition to axisymmetry may be close to $10^{-2}$ in models with a fairly wide range of geometries and kinematics.

Most of the black holes in Figure 9 fall short of the maximum mass proposed here; in fact, the mean value of $M_{h} / M_{g}$ among the roughly ten black hole candidates is only $\sim 0.5 \%$ (Kormendy \& Richstone 1995). While this fact does not invalidate our identification of $0.025 M_{g}$ as an upper limit, we would nevertheless like to understand why real black holes tend to be smaller. Following are a number of possible explanations.

1. A gradual transition to axisymmetry takes place in our simulations even for black hole masses below the critical value (Figure 7). A sufficiently slowly-growing black hole would therefore be expected to shut off its supply of fuel before accreting the full $\sim 2 \%$ of its galaxy's mass. "Slowly growing" here means that the black hole gains mass on a timescale long compared to the orbital period at the radius which is supplying most of the fuel. However, it might be difficult to reconcile such small growth rates with the short observed lifetimes of quasars.

2. Real galaxies are not structurally identical to our $N$-body models; in particular, they often have stellar envelopes in which the density falls off more slowly than the $\rho \sim r^{-3.5}$ dependence of our models. Adding stars at large radii would increase $M_{g}$ without, presumably, strongly influencing the response of the inner regions to the black hole.

3. Galaxy mergers at the current epoch might tend to reduce the ratio of black hole mass to bulge mass, by converting gas to stars and disks to spheroids. (Galaxy-galaxy interactions might also cause black holes to grow by triggering infall of gas into the nucleus.)

4. While triaxial or barlike distortions may be necessary for the efficient growth of black holes, they are probably not sufficient. Transport of stars or gas into the nucleus requires a reduction in orbital angular momentum of roughly nine orders of magnitude; gravitational torques from large-scale bars can only account for one or two of these. 
The gas-dynamical processes that are responsible for removing the remaining angular momentum are poorly understood (Osterbrock 1993). The fact that most barred spiral galaxies do not exhibit significant nuclear activity suggests that even stellar systems with strongly non-axisymmetric distortions may fail to channel mass into the nucleus.

If observed black holes are close to "maximal," their host galaxies should be nearly axisymmetric. One can hope to falsify this prediction by looking for evidence of minor axis rotation or isophote twists in the galaxies with the largest black holes. None of the galaxies in Figure 9 show such signatures. The most extensively modelled galaxy in Figure 9 is M32, which is very well described by axisymmetric models (van der Marel et al. 1997). In general, however, the evidence for significant triaxiality among early-type galaxies is weak (Statler 1995; Merritt 1997a).

Nuclear black holes may have acquired most of their mass in environments very different from their current ones, before the epoch of galaxy formation (Rees 1992). If massive black holes predate galaxies, the mechanism discussed here would not have been effective at limiting their masses. The discovery of nuclear black holes containing much more than $2 \%$ the mass of their host galaxies would lend support to the view that black holes acquired most of their mass before galaxies formed.

This work was supported by NSF grants AST 93-18617 and AST 96-17088 and by NASA grant NAG 5-2803. We thank J. Sellwood, M. Valluri and H. Zapolsky for useful discussions and for comments that improved the presentation. 


\section{REFERENCES}

Aarseth, S. J. 1994, in Galactic Dynamics and N-Body Simulations, eds. G. Contopoulos \& L. Vlahos (Berlin: Springer), 277

Adams, T. F. 1977, ApJ Suppl 33, 19

Aguilar, L. A. \& Merritt, D. 1990, ApJ, 354, 33

Barnes, J. E. \& Hernquist, L. 1996, ApJ, 471, 115

Binney, J. J. 1980, MNRAS, 190, 873

Bower, G. A. et al. 1997, preprint

Cannizzo, J. K. \& Hollister, T. C. 1992, ApJ, 400, 58

Dubinski, J. 1993, ApJ, 431, 617

Fridman, T. \& Merritt, D. 1997, Rutgers Astrophysics Preprint Series No. 208

Gebhardt, K. et al. 1996, AJ, 112, 105

Gerhard, O. E. \& Binney, J. J. 1985, MNRAS, 216, 467

Gerhard, O. E. 1987, in Structure and Dynamics of Elliptical Galaxies, ed. T. de Zeeuw (Dordrecht: Reidel), 241

Goodman, J. \& Binney, J. 1984, MNRAS, 207, 511

Goodman, J. \& Schwarzschild, M. 1981, ApJ, 245, 1087

Green, P. J. \& Silverman, B. W. 1994, Nonparametric Regression and Generalized Linear Models (London: Chapman \& Hall)

Hasan, H. \& Norman, C. 1990, ApJ, 361, 69

Hasan, H., Pfenniger, D. \& Norman, C. 1993, ApJ, 409, 91

Hernquist, L. \& Ostriker, J. 1992, ApJ, 386, 375

Kandrup, H. E. \& Mahon, M. E. 1994, Phys. Rev. E, 49, 3735

Kormendy, J. 1992, in Testing the AGN Paradigm, eds. S. S. Holt, S. G. Neff \& C. M. Urry (New York: AIP), 23 
Kormendy, J. et al. 1996, ApJ, 459, L57

Kormendy, J. et al. 1997, Univ. Hawaii Preprint IfA-97-20

Kormendy, J. \& Richstone, D. O. 1995, ARA\&A, 33, 581

Lichtenberg, A. J. \& Lieberman, M. A. 1983, Regular and Stochastic Motion (Berlin: Springer), 213

Lynden-Bell, D. 1963, The Observatory, 932, 23

Macchetto, F., Marconi, A., Axon, D. J., Capetti, A., Sparks, W. \& Crane, P. 1997, Space Telescope Science Institute Preprint Series No. 1163

Merritt, D. 1996, AJ, 111, 2462

Merritt, D. 1997a, in The Second Stromlo Symposium: The Nature of Elliptical Galaxies, A.S.P. Conf. Ser. Vol. 116, eds. M. Arnaboldi, M., G. S. Da Costa \& P. Saha (Provo: ASP), 32

Merritt, D. 1997b, ApJ, 486, 102

Merritt, D. \& Fridman, T. 1995, A. S. P. Conf. Ser. Vol. 86, Fresh Views of Elliptical Galaxies, ed. A. Buzzoni, A. Renzini \& A. Serrano (Provo: Astronomical Society of the Pacific), 13

Merritt, D. \& Fridman, T. 1996, ApJ, 460, 136

Merritt, D. \& Oh, S.-P. 1997, AJ, 113, 1279

Merritt, D. \& Valluri, M. 1996, ApJ, 471, 82

Miyoshi, M., Moran, J., Herrnstein, J., Greenhill, L, Nakai, N., Diamond, P. \& Inoue, M. 1995, Nature, 373, 127

Norman, C., May, A. \& van Albada, T. 1985, ApJ, 296, 20

Norman, C. A., Sellwood, J. A. \& Hasan, H. 1996, ApJ, 462, 114

Norman, C. A. \& Silk, J. 1983, ApJ, 266, 502

Osterbrock, D. E. 1993, ApJ, 404, 551

Peebles, P. J. E. 1972, Gen. Rel. Grav., 3, 63 
Pfenniger, D. \& Friedli, D. 1991, A\& A, 252, 75

Quinlan, G. D., Hernquist, L. \& Sigurdsson, S. 1995, ApJ, 440, 554

Rees, M. 1992, in Physics of Active Galactic Nuclei, eds. W. J. Duschl \& S. J. Wagner (Berlin: Springer), 662

Schwarzschild, M. 1981, in The Structure and Evolution of Normal Galaxies, eds. S. M. Fall \& D. Lyden-Bell (Cambridge: CUP), 43

Sellwood, J. A. \& Moore, E. 1998, in preparation

Shlosman, I., Begelman, M. C. \& Frank, J. 1990, Nature, 345, 679

Sigurdsson, S., Hernquist, L. \& Quinlan, G. D. 1995, ApJ, 446, 75

Simkin, S., Su, H. \& Schwarz, M. P. 1980, ApJ, 237, 404

Soltan, A. 1982, MNRAS, 200, 115

Statler, T. S. 1995, in Fresh Views of Elliptical Galaxies, A.S.P. Conf. Ser. Vol. 86, eds. A. Buzzoni, A. Renzini \& A. Serrano) (Provo: ASP), 27

Udry, S. 1993, A\& A, 268, 35

Udry, S. \& Pfenniger, D. 1988, A\& A, 198, 135

van der Marel, R. P., de Zeeuw, P. T., Rix, H. W. \& Quinlan, G. D. 1997, Nature, 385, 610

Voit, G. M. \& Shull, J. M. 1988, ApJ, 331, 197

Wahba, G. 1990, Spline Models for Observational Data (Philadelphia: SIAM)

Young, P. 1980, ApJ,242, 1232

This preprint was prepared with the AAS LATEX macros v4.0. 
Fig. 1.-

Evolution of the axis ratios of the initial model in the absence of a black hole. Solid lines: $b / a$ and $c / a$; dashed lines: triaxiality index, $T=\left(a^{2}-b^{2}\right) /\left(a^{2}-c^{2}\right)$. The numbers in the lower left corner of each frame are the fraction of particles, ranked by binding energy, that were used in computing the axis ratios.

Fig. 2.-

The black hole mass as a function of time (Eq. 2).

Fig. 3.-

(a)-(c) Spherically-averaged properties of the initial model (thin lines) and final models (heavy lines) in the integrations with $t_{\text {grow }}=15$, for $M_{h}=0.003,0.1$ and 0.3 . The leftward extent of the curves increases with $M_{h}$. (a) Space density; reference line has a logarithmic slope of -2 . (b) One-dimensional velocity dispersion; reference line has a logarithmic slope of $-1 / 2$. (c) Anisotropy parameter, $\beta=1-\sigma_{t}^{2} / \sigma_{r}^{2}$. (d) Projected velocity dispersion, as seen from the direction of the intermediate axis; reference line has a logarithmic slope of $-1 / 2$.

Fig. 4.-

Evolution of the axis ratios of the $N$-body models for $t_{\text {grow }}=15$. (a) $M_{h}=0.003$; (b) $M_{h}=0.01$; (c) $M_{h}=0.03$. Solid lines: $b / a$ and $c / a$; dashed lines: triaxiality index, $T=\left(a^{2}-b^{2}\right) /\left(a^{2}-c^{2}\right)$. The numbers in the lower left corner of each frame are the fraction of particles, ranked by binding energy, that were used in computing the axis ratios.

Fig. 5.-

Contours of the surface mass density as seen from the direction of the intermediate axis. Left column: initial model; right column: final time step $(t=80)$ of the model with $M_{h}=0.01$. Contours are separated by 0.3 dex in the top row, and by 0.5 dex in the bottom two rows. The heavy ellipse on the left has an axis ratio of 0.45 ; the ellipse on the right has an axis ratio of 0.6 .

Fig. 6.-

Evolution of the axis ratios of the $N$-body models with $M_{h}=0.03$. (a) $t_{\text {grow }}=45$; (b) $t_{\text {grow }}=5$; (c) $t_{\text {grow }}=0.1$. Solid lines: $b / a$ and $c / a$; dashed lines: triaxiality index, $T=\left(a^{2}-b^{2}\right) /\left(a^{2}-c^{2}\right)$. The numbers in the lower left corner of each frame are the fraction of particles, ranked by binding energy, that were used in computing the axis ratios. 
Fig. 7.-

Evolution of the intermediate-to-long axis ratio $b / a$ defined by the most-bound $50 \%$ of the stars, for $M_{h}=0.003,0.01,0.03$. The growth time of the black hole was $t_{\text {grow }}=15$ for the two smaller values of $M_{h}$, and $t_{\text {grow }}=5$ for $M_{h}=0.03$.

Fig. 8.-

Test-particle integrations in a triaxial model with a smooth core (Eq. 4) and a central point mass containing a fraction $M_{h}$ of the total mass of the model. The $z$-axis is the short axis of the figure. Each of the five orbits is defined by its starting point on the equipotential surface, which remained fixed in angular position as $M_{h}$ was increased. Orbits were integrated for approximately 10 full oscillations.

Fig. 9.-

Black hole masses $M_{h}$, in units of the solar mass, and mass fractions $M_{h} / M_{g}$ for six galaxies with securely detected black holes and large black hole mass fractions Kormendy 1992; Miyoshi et al. 1995; Kormendy et al. 1996; Bower et al. 1997; Macchetto et al. 1997; van der Marel et al. 1997). $M_{g}$ is defined as the mass of the stellar bulge in the disk galaxies (NGC 3115, NGC 4258), and as the total stellar mass in the elliptical galaxies (M32, M84, M87, NGC 3377). The dashed line is the proposed upper limit to $M_{h} / M_{g}$. 
Table 1: Properties of the $N$-Body Models

\begin{tabular}{ccccc}
\hline & Initial & & $M_{h}$ & \\
Parameter & Model & 0.003 & 0.01 & 0.03 \\
\hline$M_{\text {bnd }}{ }^{1}$ & 0.89 & & & \\
$T_{\text {max }}{ }^{2}$ & & 160 & 80 & 40 \\
$\epsilon^{3}$ & - & $4.7 \times 10^{-5}$ & $1.6 \times 10^{-4}$ & $4.7 \times 10^{-4}$ \\
$r_{h}{ }^{4}$ & - & $2.1 \times 10^{-3}$ & $6.9 \times 10^{-3}$ & $2.1 \times 10^{-2}$ \\
$r_{2}{ }^{5}$ & 0.046 & 0.041 & 0.034 & 0.023 \\
$r_{10}$ & 0.11 & 0.10 & 0.094 & 0.080 \\
$r_{50}$ & 0.48 & 0.47 & 0.45 & 0.43 \\
$T_{2}{ }^{6}$ & 0.43 & 0.37 & 0.27 & 0.15 \\
$T_{10}$ & 0.70 & 0.64 & 0.58 & 0.45 \\
$T_{50}$ & 2.92 & 2.84 & 2.71 & 2.50 \\
\hline
\end{tabular}

${ }^{1}$ Total mass of stars remaining bound to the initial model

${ }^{2}$ Integration time

${ }^{3}$ Softening radius of the black hole (eq. 3 )

${ }^{4} r_{h}=G M_{h} / \sigma_{o}^{2}$, where $\sigma_{0}$ is the one-dimensional central velocity dispersion of the initial model

${ }^{5} r_{N}=$ spherical radius containing $N \%$ of the total mass, measured at $t=40$ in the models with nonzero $M_{h}$

${ }^{6} T_{N}=$ orbital period at radius $r_{N}$

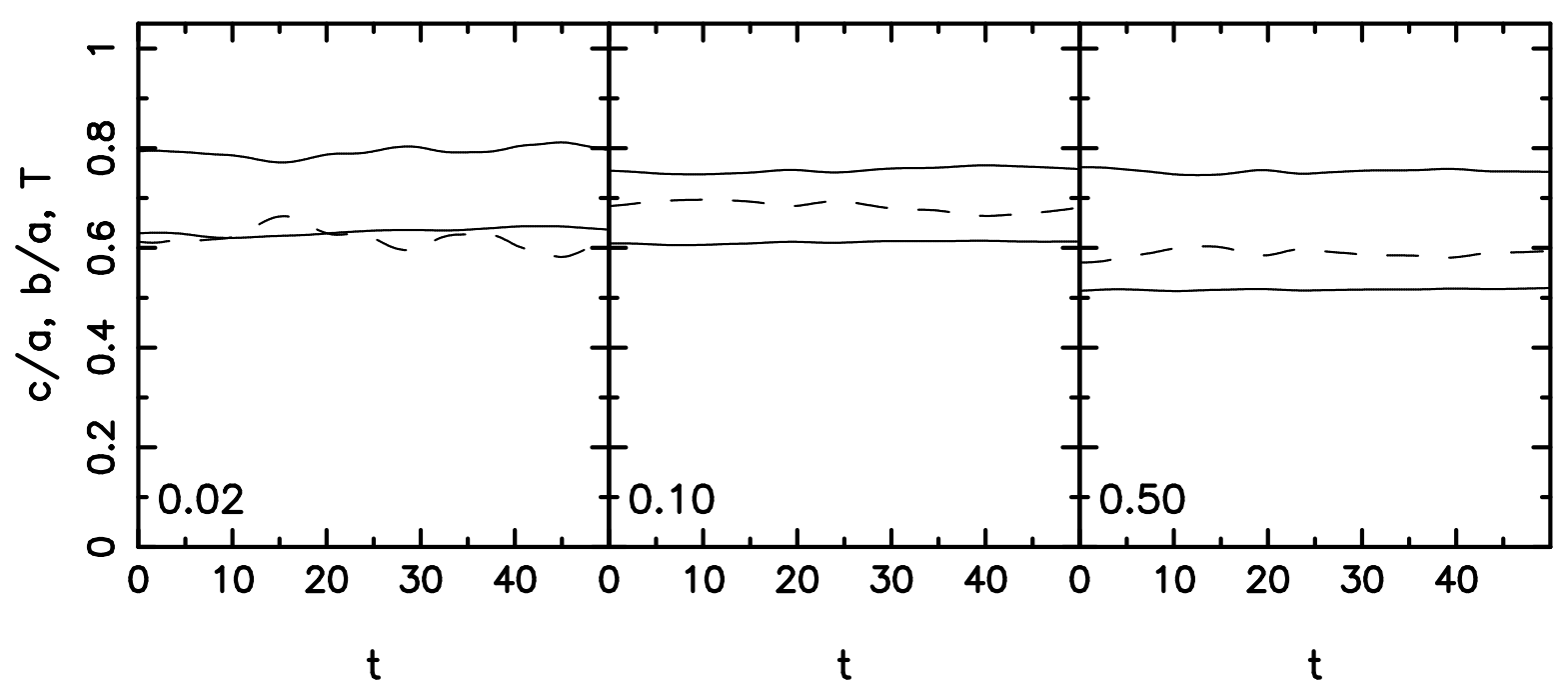

Fig. 1.- 


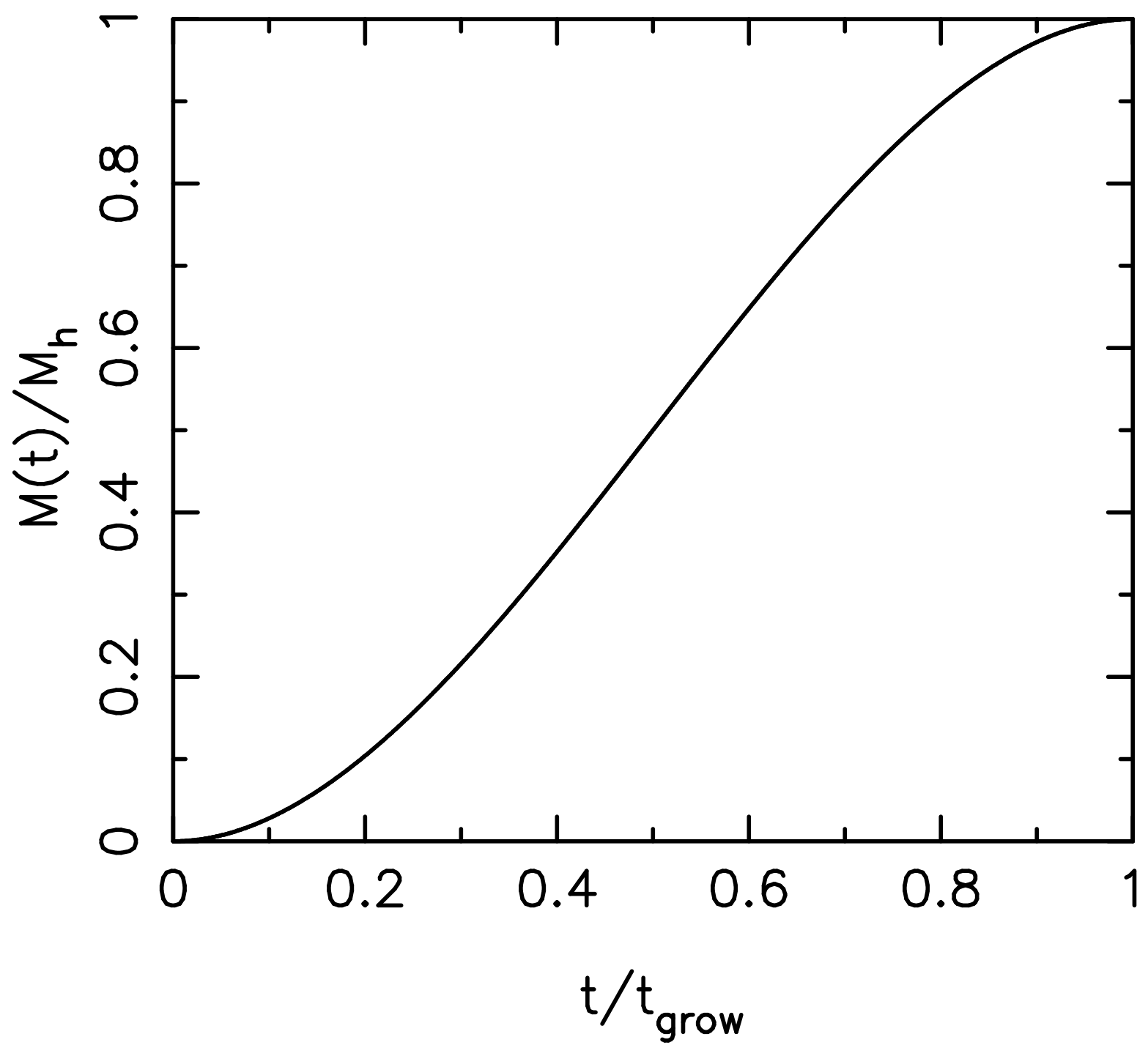

Fig. 2.- 

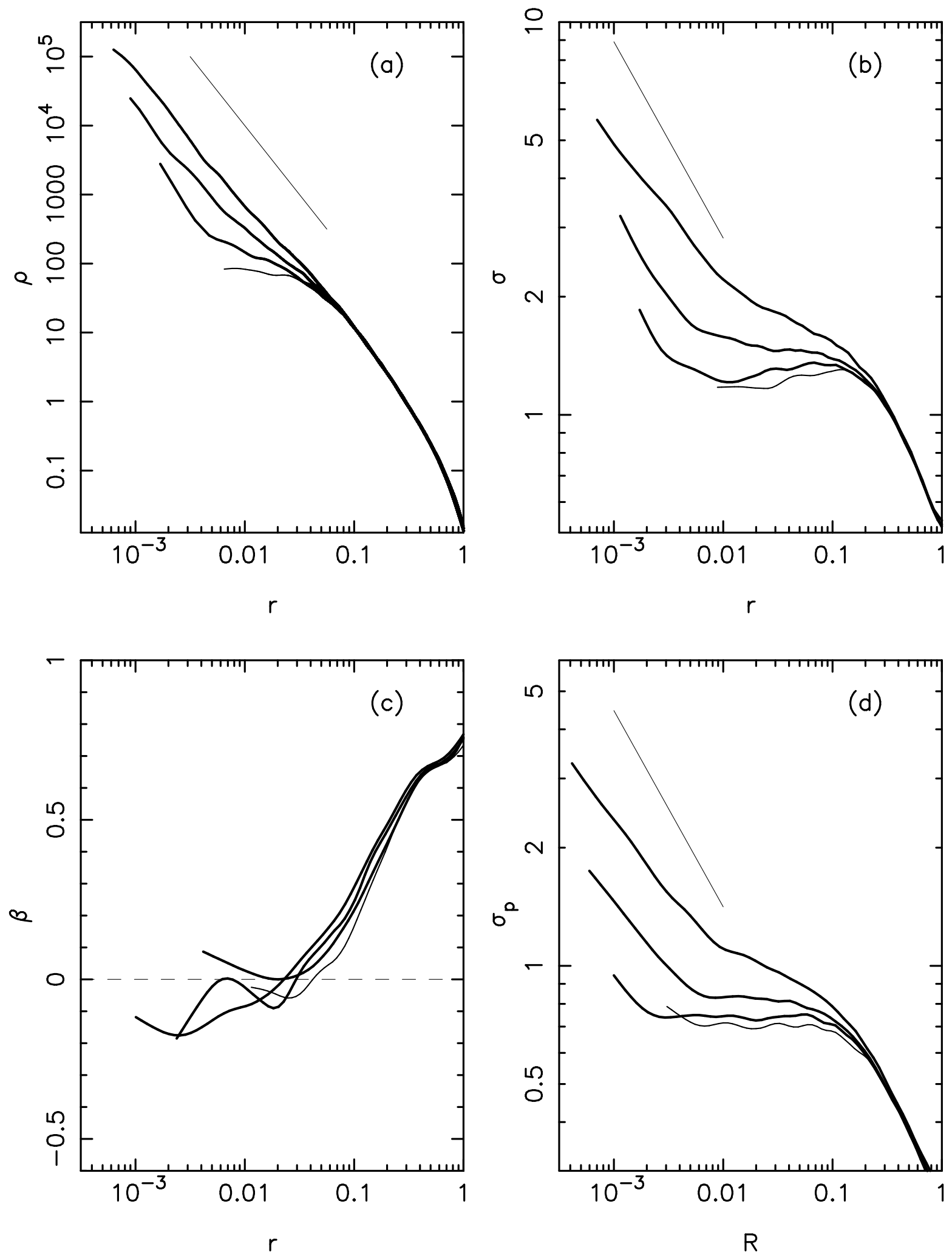

Fig. 3.- 
(a)
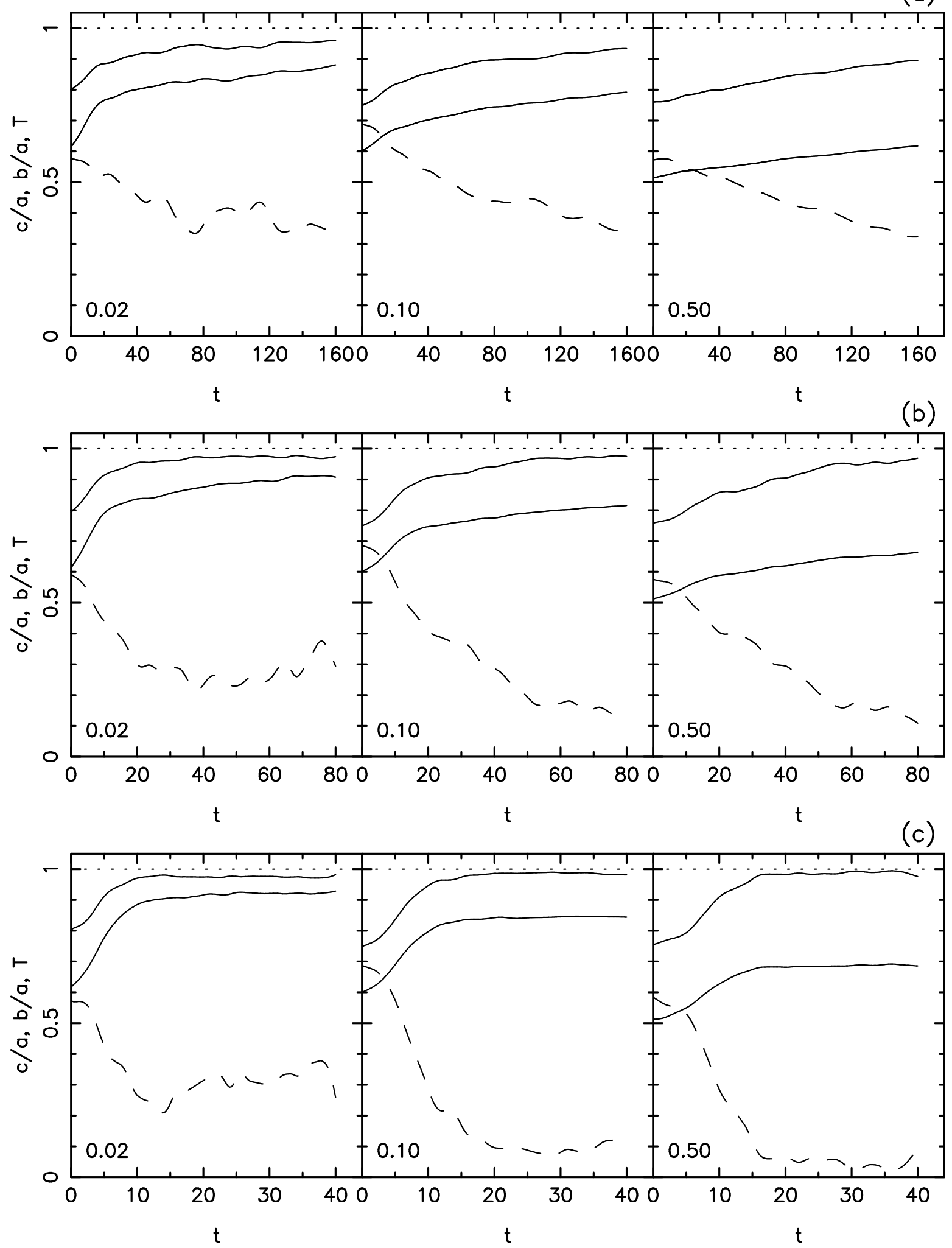

Fig. 4.- 

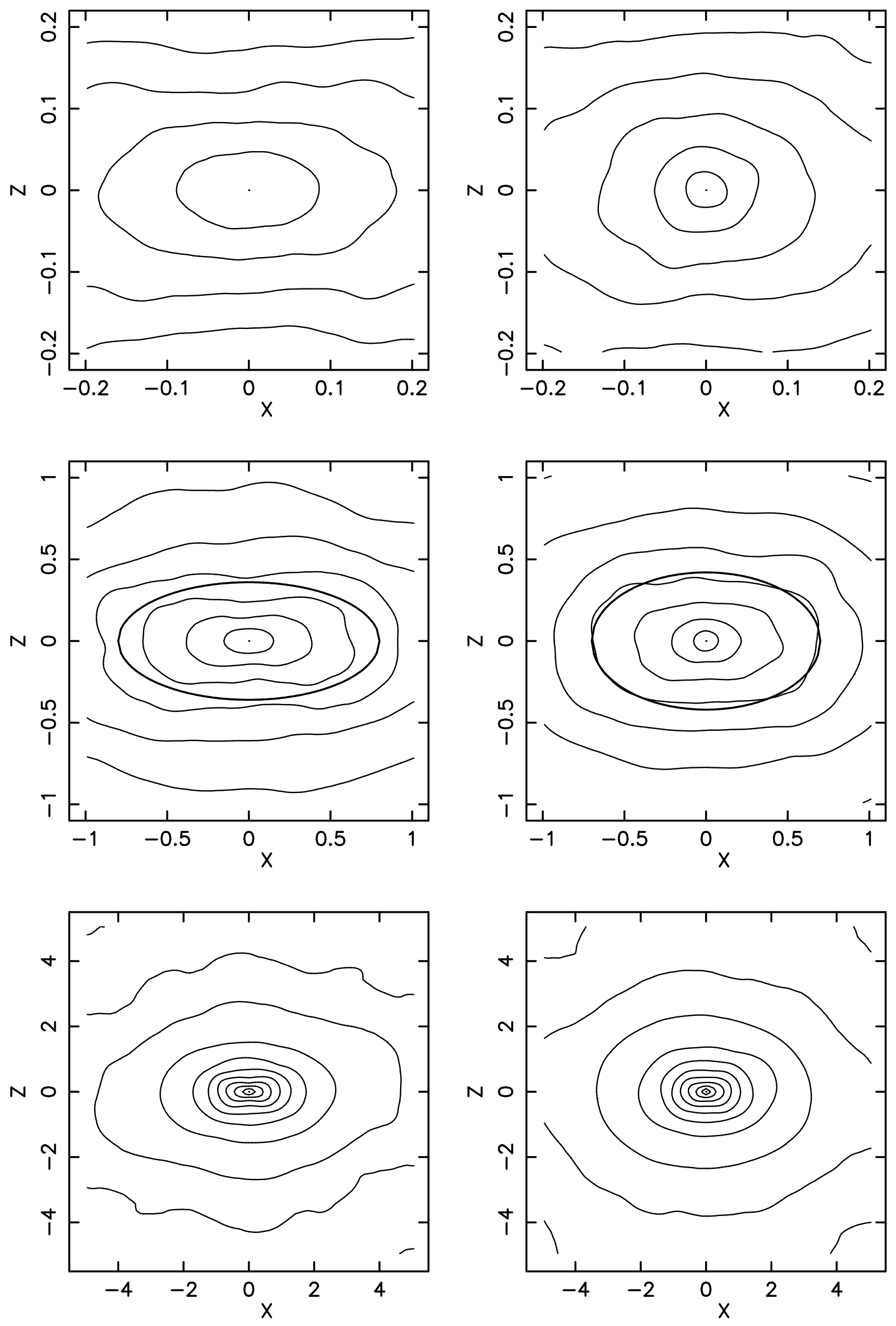
(a)

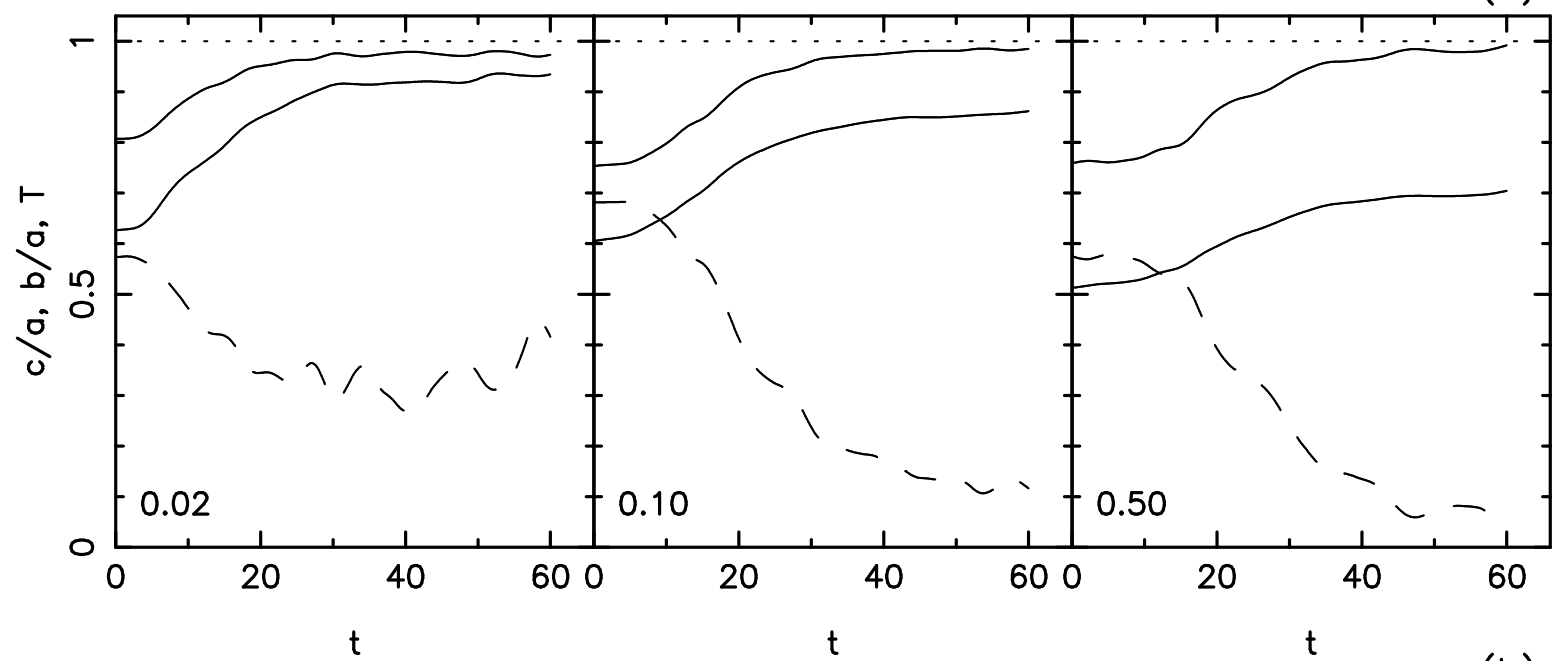

(b)
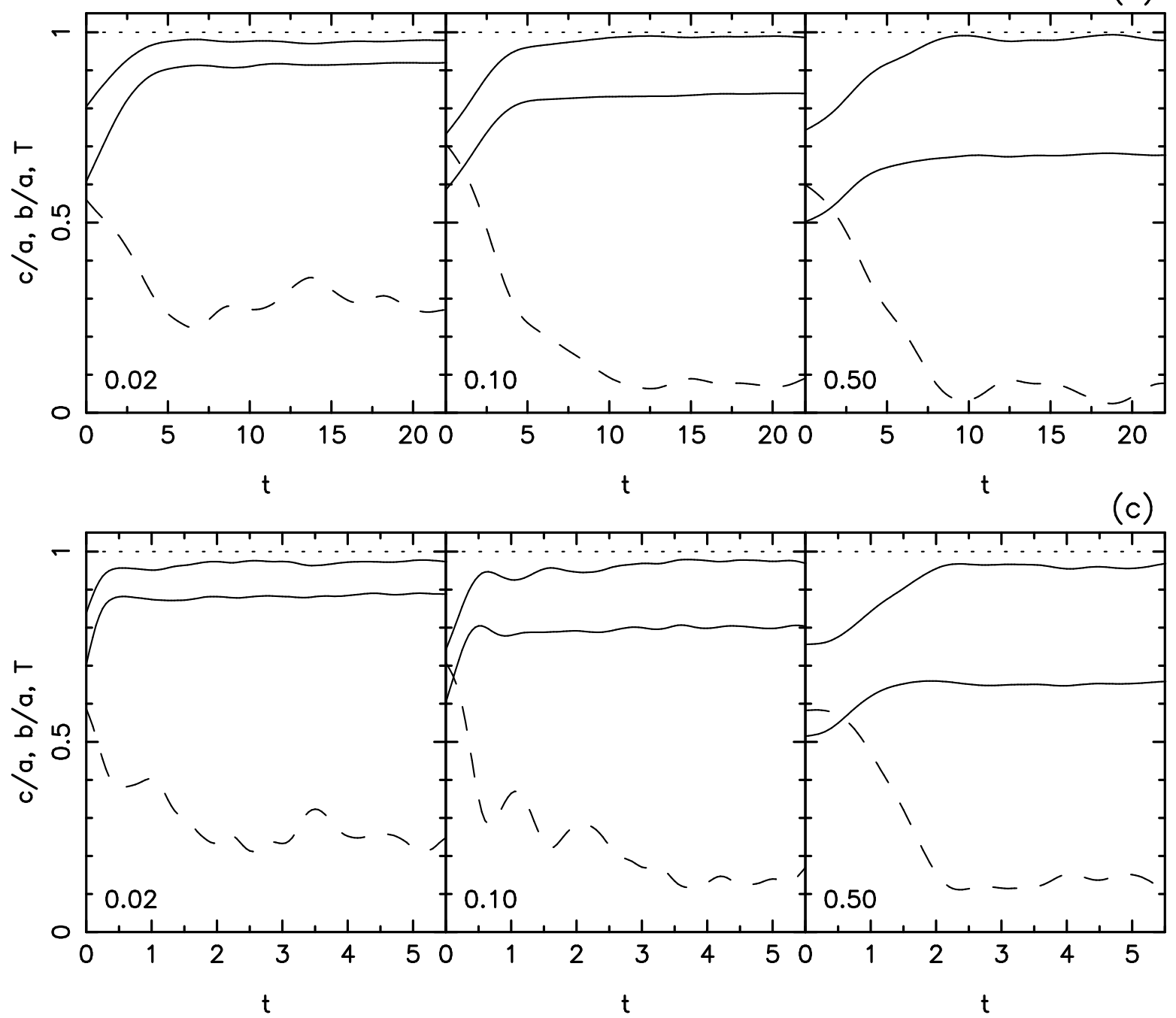

Fig. 6.- 


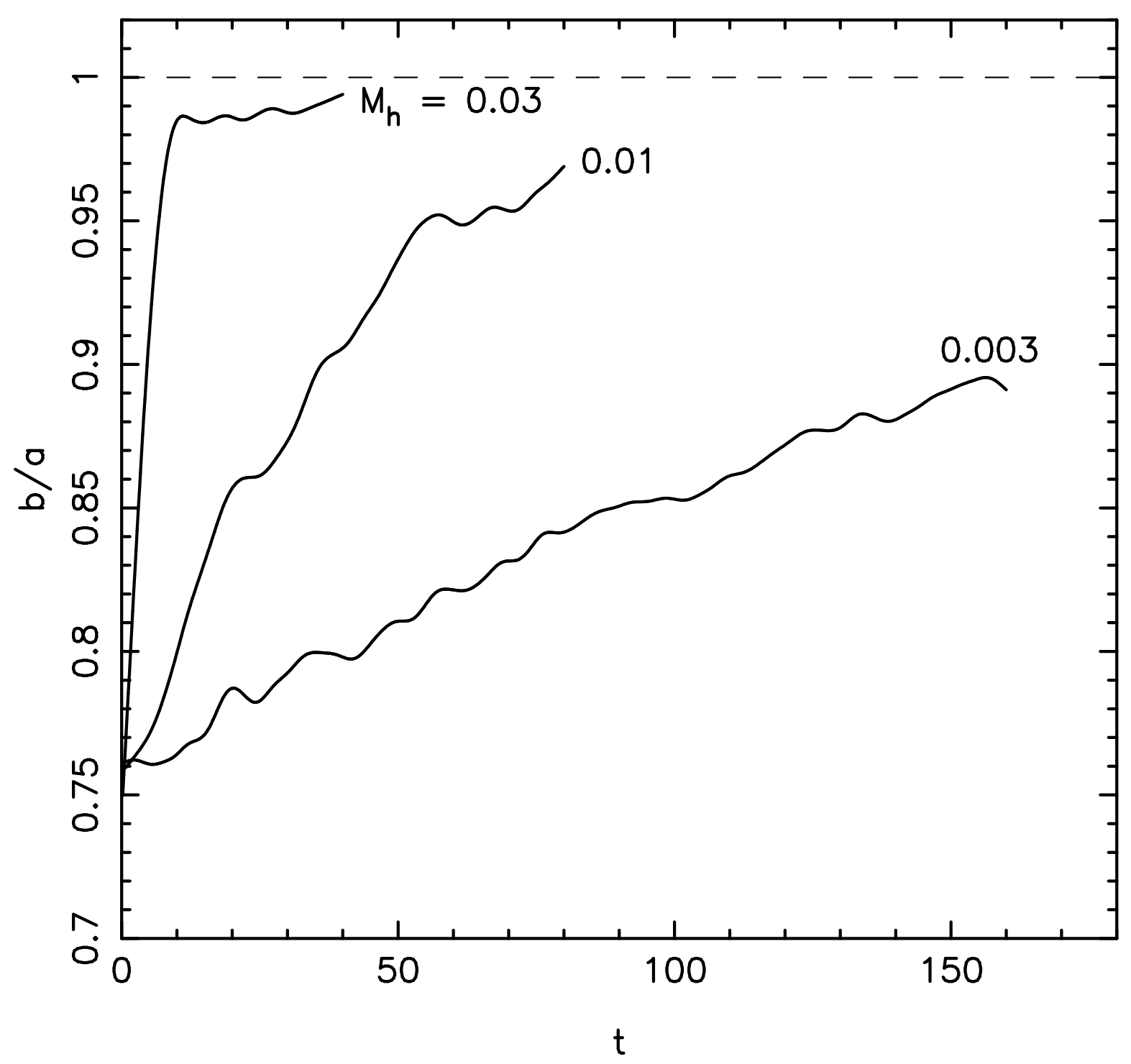

Fig. 7.- 


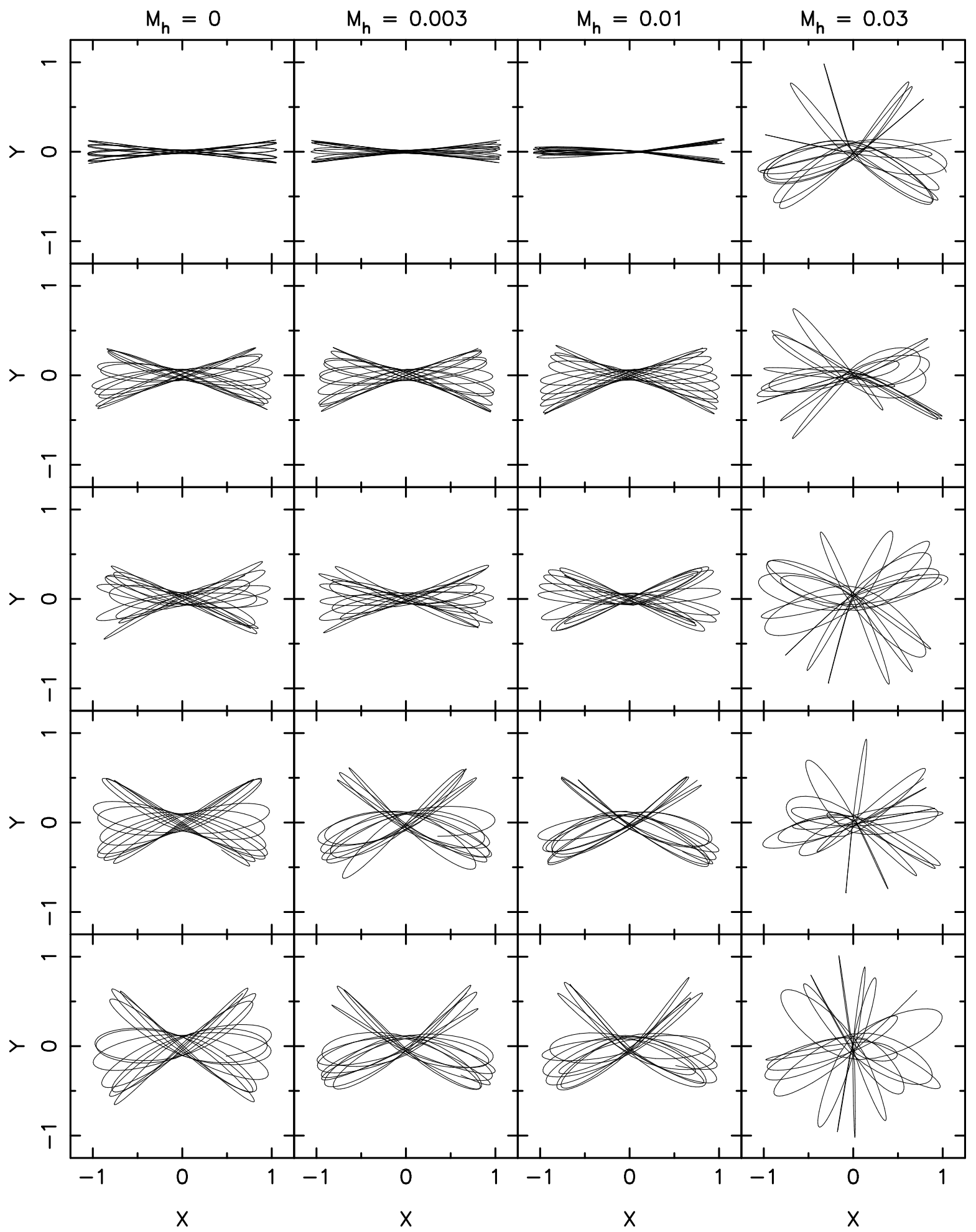

Fig. 8.- 


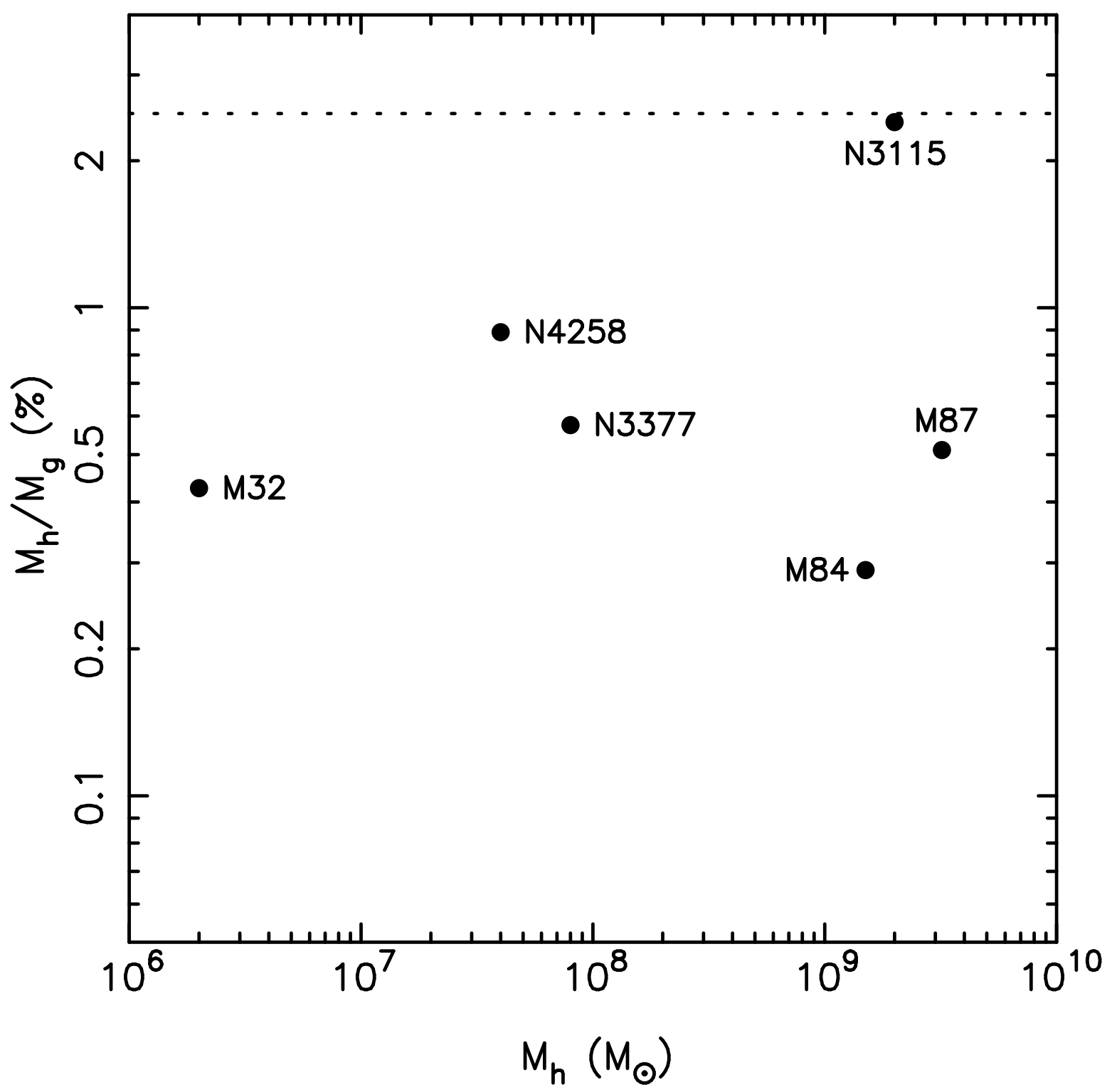

Fig. 9.- 